\title{
LA ASOCIACIÓN SIMBÓLICA ENTRE LA SALAMANDRA, CLEÓN Y LOS PESCADORES DE ANGUILAS EN LOS EMBLEMATA (1596) DE DENIS LEBEY[a]
}

\author{
THE SYMBOLIC ASSOCIATION BETWEEN SALAMANDER, \\ CLEON AND EEL FISHERMEN IN THE EMBLEMATA (1596) \\ OF DENIS LEBEY
}

\author{
Beatriz Antón Martínez* \\ Universidad de Valladolid
}

\begin{abstract}
RESUMEN. Este trabajo analiza el emblema XXIII, In Cleones nostri saeculi, qui nisi turbatis rebus laterent, del jurista y poeta francés Denis Lebey (Emblemata, 1596), un sugestivo tríptico icónico elaborado con motivos de la zoología (la salamandra), la historia antigua (el demagogo Cleón) y las actividades humanas (los pescadores de anguilas). Se identifican las principales fuentes del argumento (las Parabolae, sive Similia de Erasmo y los Collectanea de Jacobus Manlius) y también se señala la procedencia de todos los loci communes que conforman la paraphrasis que acompaña al emblema. Por último, este estudio incluye un epílogo sobre el 'Cleón' más famoso e influyente de nuestro tiempo, Donald Trump, un paralelismo percibido por numerosos estudiosos.

PALABRAS CLAVE. Emblemática, Denis Lebey, salamandra, Cleón, pescadores de anguilas.

ABSTRACT. This paper analyses the emblem XXIII, In Cleones nostri saeculi, qui nisi turbatis rebus laterent, by French jurist and poet Denis Lebey (Emblemata, 1596), a suggestive iconic triptych prepared with motifs of zoology (the salamander), ancient history (the demagogue Cleon), and human activities (the eel fishermen). The main sources of the argument are identified (the Parabolae, sive Similia of Erasmus and the Collectanea of Jacobus Manlius) and the authorship of all loci communes that comprise the paraphrasis that accompany the emblem is also indicated. Finally, this study includes an epilogue on the most famous and influential 'Cleon' of our time, Donald Trump, a parallelism perceived by many scholars.
\end{abstract}

KEYWORDS. Emblematics, Denis Lebey, Salamander, Cleon, Eel fishermen.

[a] Este artículo se inserta en el «Proyecto de Investigación del Plan Nacional de I+D PGC2018-094604-B-C31 (MCIU / AEI / FEDER, UE). This paper is part of the Research Project of the National R\&D Plan PGC2018094604-B-C31 (MCIU / AEI / FEDER, UE).

Agradezco a los profesores Francisco J. Talavera Esteso (Universidad de Málaga), Ángel C. Urbán Fernández (Universidad de Córdoba) y Carlos E. Pérez González (Universidad de Burgos), habituales lectores de mis trabajos, su revisión siempre enriquecedora.

* Correspondencia a / Correspondence to: Beatriz Antón Martínez, Universidad de Valladolid, Facultad de Filosofía y Letras, Departamento de Filología Clásica, Plaza del Campus, s/n (47011 Valladolid) - beatriz@fyl.uva.es - https://orcid.org/0000-0003-3050-2733.

Cómo citar / How to cite: Antón Martínez, Beatriz (2021), «La asociación simbólica entre la salamandra, Cleón y los pescadores de anguilas en los Emblemata (1596) de Denis Lebey», Veleia, 38, 251-268. (https://doi.org/10.1387/veleia.21655).

Recibido: 15 abril 2020; aceptado: 27 octubre 2020.

ISSN 0213-2095 - eISSN 2444-3565 / (C) 2021 UPV/EHU

(c) (i) $\Theta$ Esta obra está bajo una licencia

Creative Commons Atribución-NoComercial-SinDerivadas 4.0 Internacional 
El género emblemático alcanzó su momento álgido en Francia en el siglo Xvi ${ }^{1}$, convirtiéndose en una de las manifestaciones literarias más sofisticadas y seductoras merced a la labor conjunta de autores $^{2}$, traductores ${ }^{3}$ y comentaristas ${ }^{4}$, impresores / libreros ${ }^{5}$ y artistas grabadores ${ }^{6}$. Corona esta fecunda aetas aurea el poeta y juez calvinista Denis Lebey (Troyes, 1551-Metz, 1607) ${ }^{7}$, coevo de los reyes Enrique III y Enrique IV; por consiguiente, vivió una época convulsa en lo religioso (guerras entre católicos y hugonotes) y en lo político (Guerra de los Tres Enriques por el trono francés), con masacres y motines populares que inevitablemente dejaron su impronta en la colección de emblemas que compuso en sus horae subsecivae, pues el studium emblematicum se había convertido en un otium asociado a la ciencia jurídica desde el dux et praeses Andrea Alciato (Antón 2016).

Lebey poseía una sólida formación: estudió litterae humaniores (en Ginebra fue discípulo de Pierre de la Ramée) y jurisprudencia (entre sus maestros estaba Jacques Cujas, corifeo del humanismo jurídico junto con Alciato). Huyendo de las disensiones políticas y religiosas recaló en Metz (1589), donde católicos y protestantes convivían en un clima de aparente concordia, y engrosó el coetus eruditorum de dicha ciudad, caracterizado por su adhesión a la Iglesia Reformada, el cultivo de la poesía, la pasión por la antigüedad clásica, el espíritu de tolerancia y la adscripción a las nuevas corrientes filosóficas, la neoestoica en particular.

En ese savant ambiente religioso e intelectual compuso sus Emblemata, que tuvieron dos ediciones (Antón 2014, 128-137): la primera apareció en 1596 (Fráncfort, De Bry) y su principal modelo son los Emblemata (Fráncfort, De Bry, 1593) de su amigo, el anticuario y poeta Jean Jacques Boissard $^{8}$; consta de 63 emblemas, con elegantes picturae diseñadas por el propio Boissard y calcografiadas por De Bry; cada emblema está acompañado de una paraphrasis o explanación en prosa, que le encargó el impresor De Bry (Antón 2014, 131), elaborada a base de excerpta de cuya autoría muy rara vez informa9. La segunda edición, que vio la luz en 1600, en Heidelberg (conocida como editio Voegeliniana), reúne 226 emblemata nuda y carece de paraphrasis.

El libro de Lebey, fruto de una estrecha colaboración con Boissard, es un inagotable filón clásico y apotegmático. La mayoría de sus argumentos explotan la historia natural y los personajes, divinidades, mitos y anécdotas del mundo greco-romano, para exponer simbólicamente conceptos filosóficos, políticos, religiosos y literarios. Habida cuenta de que nada había en el theatrum vitae humanae que no fuera susceptible de una lectura emblemática, i. e., simbólica, se impone averiguar a qué aspectos superiores de la vida o a qué realidad concreta remite el emblema XXIII, un sugestivo tríptico icónico que articula motivos tan dispares como la zoología (la salamandra), la historia antigua (Cleón) y las actividades humanas (los pescadores de anguilas). Para ello, analizaré tanto la

\footnotetext{
1 Véanse Russell 1985; Saunders 1988; Adams, Rawles \& Saunders 1999-2002; Graham 2001; Adams 2007.

2 Tanto de libros de emblemas en francés (G. de la Perrière, M. Scève, G. Corrozet, G. Gueroult, C. Paradin, G. de Montenay, etc.) como en latín (P. Coustau, B. Aneau, Th. de Bèze, J. J. Boissard, etc).

3 Del Emblematum Liber de Alciato (J. Lefevre, B. Aneau) y de otros libros de emblemas latinos (P. Coustau, J. Sambucus, H. Junius, etc.).

4 B. Aneau y C. Mignault escribieron comentarios al Emblematum Liber de Alciato.

5 En París: Ch. Wechel (allí en activo desde 1522), D. Jenot, J. de Marnef, J. Richer; en Lyon: J. Moderne, J. de Tournes, G. Rouille, M. Bonhomme, etc.
}

6 B. Salomon, P. Woeriot, P. Eskrich, etc., aunque no siempre es fácil identificar los nombres de los diseñadores y grabadores de las picturae.

7 Sobre Lebey y su obra emblemática, véanse Choné 2007; Antón 2014, 2019a/b; Meerhoff 2017.

8 Para la relación entre ellos y sus respectivos libros de emblemas, véase Antón 2014, 121-128, 134-138.

9 Nombra una veintena de autores clásicos (Homero, Heródoto, Píndaro, Esquilo, Eurípides, Platón, Aristóteles, Enio, Catón, Cicerón, Virgilio, Horacio, Jerónimo, Ambrosio...) en poco más de una docena de emblemas (n. ${ }^{\text {os }} 3,8,31,41,53,45,46,47,52,53,59$, 60, 62). 
res picta y la res significans del emblema, como la paraphrasis correspondiente. Obviamente la editio princeps será la basa sobre la que descansará el fuste de este estudio, que solo ornará puntualmente la editio Voegeliniana.

\section{EL EMBLEMA TRIPLEX}

La colección de emblemas de Lebey se ajusta al emblema triplex o canónico (inscriptio, pictura y subscriptio), en la línea del Emblematum Liber de Alciato. El emblema XXIII, In Cleones nostri saeculi, qui nisi turbatis rebus laterent ("Sobre los Cleones de nuestro tiempo, que permanecen ocultos a no ser que haya disturbios») [Fig. 1], se incardina en el nutrido grupo temático de la guerra y de la paz (n. ${ }^{\text {os }}$ XIX-XXXIV) (Antón 2014, 136) ${ }^{10}$. Lleva un dedicatario (Simon Grasso, Tricass.), Simon Grass, natural de Tricassinum (Troyes), coterráneo de Lebey.

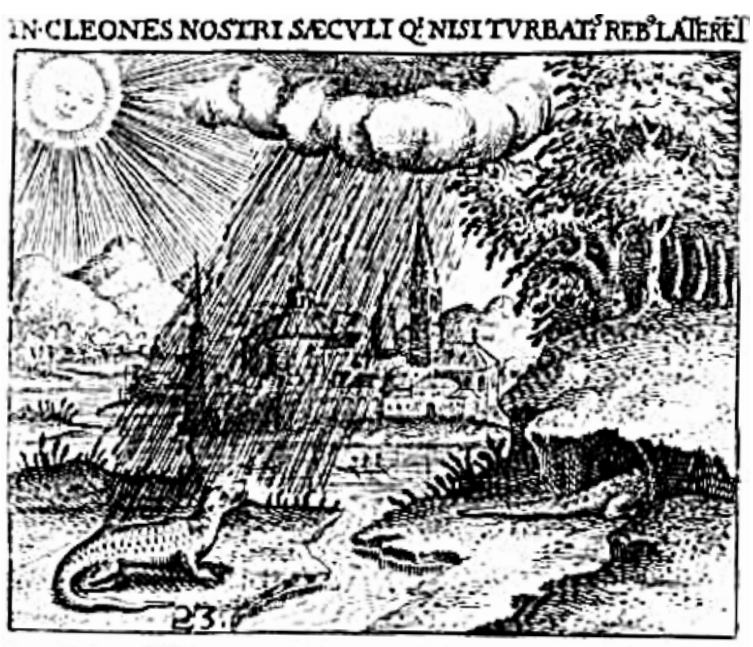

\footnotetext{
Vt quando colum nigrefcere capit al Aufrib, Et ruit effu!a furbidus iniberagus.

Catpere tum demum vitules invipit auras, Temperiecalipoll Salamardraperit.

Sic morus inter patrie, belligiprecellas Qutm nofh is for vont omrita difidïs.

Emergunt multiparsignotifsima vulgi, Sole incrum pacis quiredeunte latent.
}

Figura 1. D. Lebey, Emblemata, Fráncfort, 1596, emb. 23. URL: https://archive.org/details/dionysiilebeibatOOlebe. No copyright.

10 Otro emblema de dicho grupo, el $\mathrm{n}^{\circ}$ XXVIII, Paci studere praestat quam bello («Es mejor favorecer la paz que la guerra»), lo he estudiado recientemente (Antón 2019b). 
En la editio Voegeliniana se agrega el dato de que es Tesorero Real de Troyes (Ad Simonem Grassum, Trecass. [sic] Quaestorem Regium). No siempre es fácil establecer una conexión entre el argumento de cada emblema y el dedicatario, que suele ser un relevante personaje (la mayoría de ellos son juristas, médicos y teólogos, sin distinción entre católicos y protestantes), por lo que quizá Lebey actuaba guiado por meras razones prácticas y de variatio (Antón 2014, 135).

En la inscriptio o título (de tipo descriptivo), que desvela la res significata, y no se refleja en la pictura, se alude obviamente al demagogo ${ }^{11}$ Cleón de Atenas (ca. 460-422 a.C.) (Lafargue 2013; Saldutti 2014), que llegó a ser el principal hombre de la ciudad tras morir su rival Pericles. Las fuentes griegas primarias (Tucídides y Aristófanes, coetáneos de Cleón y abiertamente hostiles a su política) ${ }^{12}$ coinciden en mostrarlo como un personaje vil y un protervo agitador, responsable del declive ateniense. Sin embargo, ya hace tiempo que la crítica ha puesto en tela de juicio la visión sesgada que la tradición ofrecía de él e incluso ha reivindicado su figura ${ }^{13}$. Ahora bien, no es el Cleón histórico el que aquí nos interesa, sino el Cleón paradigma de los demagogos y sediciosos que transmite Lebey, secundando una ancestral y tergiversada tradición.

La pictura, en una narración visual simultánea, muestra dos escenas: en la de la izquierda se ve una salamandra bajo la lluvia, y en la de la derecha, una salamandra que se esconde en un tronco (pero el sol, que motiva la huida del caudado, se encuentra en la otra escena). La res significans, i. e. la salamandra (que constituye el motivo pictórico), se menciona en el epigrama en el cuarto verso, y no es la popular salamandra ígnea ${ }^{14}$, sino la común, según habremos de comprobar:

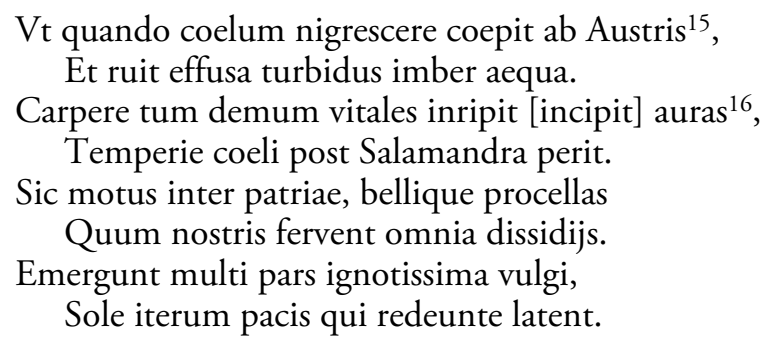

11 En la antigua Grecia el demagogo era el 'líder del pueblo', acepción que ha cambiado considerablemente desde entonces, convertido hoy en un término peyorativo (Gustainis 1991, 155).

12 Plutarco y Diodoro Sículo son testimonios tardíos $\mathrm{y}$, además, plantean problemas de fuentes. Vid. Paladini 1958; Mann 2007, 75-96, 170-190; Morwood 2009, 354-356; Foster 2017.

13 En los años 60 del siglo xx empezó a cambiar la denostada imagen que se tenía de Cleón, pero ya a mediados del siglo xIx, pace Morwood (2009, 353354), salió en su defensa G. Grote (1854). Le siguieron otros (v. gr. Busolt 1904, 1154-1157; Thibaudet 1922, 33-41); y en el siglo xxi ha salido vehementemente en su favor L. Canfora (2016), quien trata (cap. 9\$6) del «auténtico Cleón»y concluye con esta aseveración: "l'obsoleto ritratto, subalterno dei lazzi aristofanei, di un Cleone popolano-demagogo (un 'cane del popolo' da IV secolo o un Pugačëv ante litteram) è destituito di fondamento»; en otra parte (2017) vuelve sobre el tema (cap. $1 \$ 3$ ) para decir que "Cleone appartenne al ceto dei cavalieri e non fu certo il gaglioffo sguaiato la cui immagine repelente Aristofane ha trasmesso alla posterità».

$14 \mathrm{Ha}$ sido prioritariamente la salamandra ígnea la que ha despertado el interés de los estudiosos de literatura simbólica, de tratados de magia y de alquimia del Medievo y del Renacimiento. Esta salamandra, según las fuentes antiguas, podía vivir en el fuego sin consumirse; y, al contrario, por su excepcional frialdad era capaz de apagar las llamas (García Arranz 1990; Biederbick 2017, 294-303).

15 El Austro (Auster) es el viento del sur que trae abundantes lluvias (Verg. Aen. 5, 696; georg. 1, 462; Tib. 1, 1, 47; Ov. met. 2, 21; etc.).

16 Vitales auras, reminiscencia virgiliana (Aen. 1, 387). 
«Cuando el cielo comienza a oscurecerse con el soplo del Austro y una violenta tormenta se precipita en torrencial aguacero, por fin entonces la salamandra empieza a vivir y después, cuando escampa, muere. De igual modo, en medio de los tumultos de la patria y las tormentas de la guerra, cuando todo bulle debido a nuestras discordias, salen muchos, la parte más ignota del vulgo, que, al volver el sol de la paz, se ocultan de nuevo.»

El epigrama, acorde con la oración comparativa que lo conforma (ut... sic), se divide en dos partes simétricas: una zoológica (los dos primeros dísticos), en la que se describe el comportamiento natural de la salamandra (sale cuando llueve y se oculta cuando escampa; en el dibujo el sol debería estar situado a la derecha); y otra simbólica (los dos dísticos restantes), en la que el anfibio se asimila a la parte más humilde y desconocida del pueblo (pars ignotissima vulgi), por oposición a los no(ta)biles; esa pars solo hace acto de presencia en los momentos conflictivos (procellas belli) y desaparece cuando retorna la calma (Sole... pacis... redeunte).

Se detectan, no obstante, algunas divergencias en el emblema triplex: la salamandra (res significans) del dibujo y del epigrama no halla parangón en el título; y a los Cleones (res significata) del título se alude solo transversalmente en el hexámetro Emergunt... vulgi.

\section{La PARAPHRASIS}

El paratexto, cuya composición — como adelanté— le fue encomendada por De Bry y tiene su patrón en el que ilustra el Emblematum liber (1593) de Boissard ${ }^{17}$, está encabezado por el epígrafe In Cleones nostri saeculi, repitiendo solo la primera parte del título del emblema. Su contenido es susceptible de dividirse en tres apartados: el primero, muy breve, se centra en la salamandra y su simbolismo; el segundo, en los anguillarum captatores como trasunto de Cleón, icono a su vez de los demagogos y amigos de revoluciones; el tercero, elaborado centonis instar, abunda en la desdeñosa opinión sobre los demagogos.

\subsection{La salamandra y su simbolismo}

La paráfrasis se abre con la descripción de la salamandra y de su modus vivendi en términos plinianos ${ }^{18}$ : Salamandra animal est lacerti figura, stellatum, numquam nisi magnis imbribus prove-

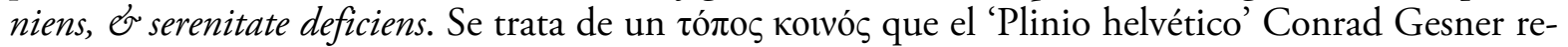
pite y amplia en sus celebérrimas Historiae animalium. Y traigo a colación al naturalista helvético porque en el Libro II (de quadrupedibus oviparis) (1554, 74-90) trata de esas salamandras que habitan lugares fríos y húmedos (loca frigida \& humida), cerca de las fuentes, en los prados y cerca de los senderos (circa fontes, in pratis \& circa semita); y, siguiendo a P. A. Matthioli ${ }^{19}$, añade que aparecen sobre todo con las lluvias de primavera o de otoño (Tempore pluuiali praecipue uerno \& autumnali), pero con el intenso calor del verano rarísima vez salen de su escondrijo (aestate calores in-

17 Es muy probable que este paratexto le fuese solicitado también a Boissard por el impresor De Bry con la intención de atraer a un público más cultivado.

18 Plin. nat. 10, 86, 188: salamandra, animal lacertae figura, stellatum, numquam nisi magnis imbribus proveniens et serenitate desinens ("la salamandra, animal con aspecto de lagarto, con motas en forma de estrella, que nunca sale a no ser con copiosas lluvias y que desaparece cuando escampa»).

19 Solo nombra al autor, sin más datos. Matthioli (1554, 197-198) trata de este anfibio en el Lib. II, cap. LVI ( $\Sigma \alpha \lambda \alpha \mu \alpha \dot{\alpha} v \delta \rho \alpha$. Salamandra), dedicando al final solo tres líneas a la salamandra aquatica. 
tensi, rarissime e terra prodeunt) $(1554,76 \mathrm{C})$, por eso verlas es anuncio de lluvias (signum pluuiae futurae) $(1554,79 \mathrm{E})$. En el vol. IV (de piscium et aquatilium animantium natura) se ocupa de la salamandra aquatilis (1558, 967-968), advirtiendo que ambos caudados, el terrestre y el acuático, se designan con el mismo nombre ('salamandra') en griego, en latín y en las lenguas romances. Así pues, la bestiola de nuestro emblema es la salamandra común ${ }^{20}$, terrestre o forestal ${ }^{21}$.

\section{Ad S.al.tm.tndrim.}

\section{In milites.}

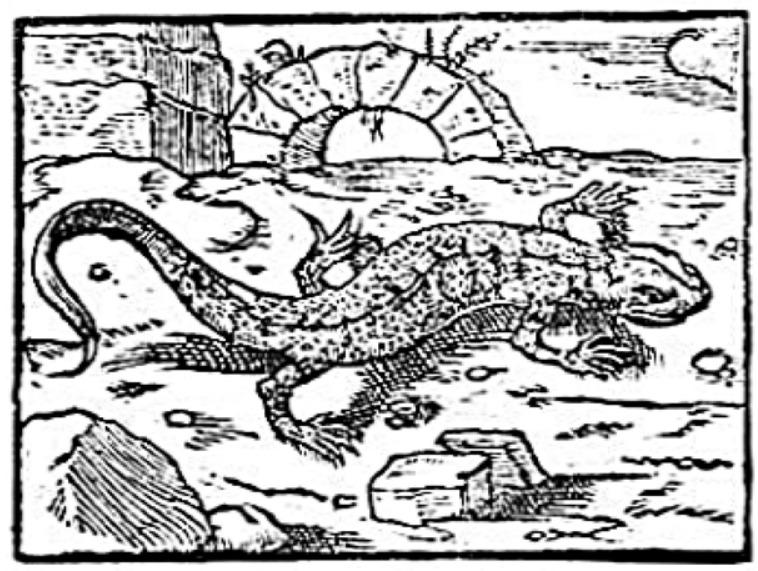

Imbrifer autumnus vitales addidit auras, Temperics fccit te Salamandra mori. Captat opes milcs tunc cum furialis Enio Euocat emeritos ad fer a bella duces: Quid fipercu!flairentur faderaporca, Pauper in exiguadeficit illc domo.

Figura 2. P. Coustau, Pegma cum narrationibus philosophicis, Lyon, 1555, pág. 95. URL: http://biblioteca.galiciana.gal/gl/consulta/registro.do?id=8693. Dominio público.

Lebey conecta el preámbulo zoológico con el comportamiento humano (los que solo salen en las insurrecciones para sacar rédito de ellas): Ita quidam non apparent, nisi statu ciuitatis bello aut seditione

20 La salamandra común (salamandra salamandra) es un anfibio sobre todo forestal, de hábitos terrestres y nocturnos (durante el día se refugia bajo la hojarasca, troncos caídos, piedras, galerías u oquedades del terreno con un alto grado de humedad); las poblaciones ovíparas (no las vivíparas) necesitan estar cerca del agua (arroyos o charcas) para la reproducción. Comienza su actividad anual con las primeras lluvias del otoño tras pasar el verano inactiva (Velo-Antón \& Buckley 2015).

21 Henkel \& Schöne (2013, 736-738) incluyen este emblema de Lebey (y los de P. Coustau y J. Camerario que mencionaré infra) bajo el epígrafe Wassersalamander ('salamandra acuática'), y también García Arranz $(1990,63)$. Sobre la salamandra acuática (tritón) y la terrestre en estos emblematistas, vid. Antón, e. p. 
turbato, ijdem rebus pace compositis, emoriuntur («Así, algunos no aparecen a no ser que perturbe la ciudad una guerra o un motín, y esos mismos, una vez pacificada la situación, se extinguen»). El origen de este símil 'político', que a juicio de Margolin $(1975,295)$ es «assez artificielle», se localiza en el Parabolarum, siue Similium Liber (1514) de Erasmo ${ }^{22}$. Igualmente, el jurista francés Pierre Coustau (Saunders 2001; Hayaert 2005; 2008), inspirándose en esta ó titulado Ad salamandram, y subtitulado In milites (1555, 95-97) [Fig. 2].

La subscriptio desentraña el doble título ${ }^{23}$ :

Imbrifer autumnus vitales addidit auras,

Temperies fecit te Salamandra mori.

Captat opes miles tunc cum furialis Enio ${ }^{24}$

Evocat emeritos ad fera bella duces:

Quod si percussa iurentur foedera porca ${ }^{25}$,

Pauper in exigua deficit ille domo.

«El pluvioso otońo, salamandra, te da la vida, pero el calor hace que mueras. Ambiciona riquezas el soldado cuando la furiosa Enío llama a los generales veteranos a entablar feroces guerras; pero si, tras golpear una puerca, se firma un tratado [de paz], ese soldado acaba pobre en una mísera casa.»

Cuando Lebey redactaba sus Emblemata, el médico alemán Joaquín Camerario trabajaba en sus Symbola et Emblemata. En la Centuria IV, concebida ex aquatilibus et reptilibus y publicada póstumamente (1604 [1605]) por su hijo Luis (Papy 2003, 212-213; Antón 2012, 158-159; e.p.; Hendrikx 2018), se dedica a la salamandra el emb. LXX, Coelo turbato alacrior («Con tiempo revuelto soy más feliz») [Fig. 3].

Tanto la subscriptio (un dístico) como el comentario confieren a la salamandra un significado político que remeda el símil de Erasmo y el pegma de Coustau, y muestra notables concomitancias con el emblema de Lebey. Por motivos de espacio, transcribo tan solo el dístico ${ }^{26}$, que — según adelanté- compuso su hijo Luis:

Sunt pluviae mihi vita, necem sed Sirius ${ }^{27}$ affert,

In pace $\&$ languent, quos fera bella fovent.

«Las lluvias me dan la vida, en cambio Sirio me causa la muerte. En la paz también languidecen esos a los que las crueles guerras alientan.»

${ }^{22}$ Margolin 1975, 294: Salamandra non nisi magnis hymbribus prouenit, serenitate deficit: ita quidam non apparent, nisi statu ciuitatis bello aut seditione turbato, iidem rebus pace compositis emoriuntur.

${ }^{23}$ En la Narratio philosophica, o discurso en prosa que ilustra el emblema (Saunders 2001, 38-39), el autor vuelve sobre esa comparación sin separarse de Erasmo (adag. III.VI.79).

${ }^{24}$ Enío ('Evvó), diosa menor de la guerra que iba en el séquito de Ares (Hom. Il. 5, 592); los romanos la identificaron con Belona.
25 Sobre este rito romano y sobre la víctima, a la que se golpeaba con una piedra de sílex (porca se usa a veces en lugar de porcus, v. gr., Verg. Aen. 8, 641), véase Gladhill 2016, 49-53, 147-148, 156, 162.

26 Este emblema de Camerario y otros suyos de significado afín son analizados en Antón (e. p.).

27 Sirius ( $\Sigma$ cípros), en la constelación Canis maior, es la estrella más brillante del cielo nocturno. Aquí designa la época más calurosa del año, los dies caniculares

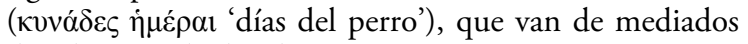
de julio a mediados de agosto. 


\section{OE L O ${ }^{\mathrm{Lx}} \mathrm{T}$ V R B A- T O ALACRIOR.}

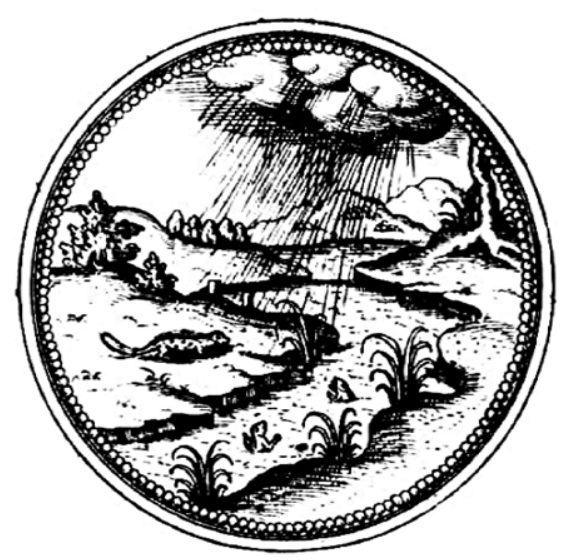

Suns pluvia milti vita, netem fed Sirius affert:
In pace do Langueit, quos fera bella fovens.

Figura 3. J. Camerario, Symbola et Emblemata. Centuria IV, [s. l.] 1604 [1605], emb. 70. URL: https://archive.org/details/ARes125022/mode/2up. No copyright.

Hasta aquí la sección concerniente a la salamandra, en la que he considerado oportuno informar, siquiera someramente, de los otros dos emblematistas que, también en el siglo Xvi, atribuyeron al urodelo una interpretación análoga a la de Lebey.

\subsection{Los pescadores de anguilas, simbolo de los Cleones de la época}

El autor continúa su explanatio con un párrafo que, conceptualmente, se vertebra en dos partes de desigual extensión (las separo en el texto mediante doble barra):

Vtque qui anguillas capiunt prius aquam turbant, ita Cleon non potest crescere nisi prius turbata Republica. Optimi quique paci ac tranquillitati Reipublicae student, sceleratissimi maximum bellum et perturbationes concupiscunt, \| quibus afflicta fides in pace, rebus turbatis alacres, et per incerta tutissimi sunt ${ }^{28}$, ut qui privatim degeneres, in publicum exitiosi, nihil spei nisi per discor-

28 Tac. hist. 1, 88: multi afflicta fides in pace, rebus turbatis alacres, et per incerta tutissimi sunt. Cf. Lips. Polit. VI, IV: quibus scilicet afflicta fides in pace, et re- bus turbatis alacres, et per incerta tutissimi sunt (Waszink 2004, 680). 
dias habent ${ }^{29}$. Quibus autem quies in seditionibus, in pace turbae sunt ${ }^{30}$, honores quos quieta Repub. desperant, perturbata consequuntur ${ }^{31}$. Quanto quis audacia promptus, tanto magis fidus, rebusque motis potior habetur. ${ }^{32}$

«Y del mismo modo que los pescadores de anguilas antes enturbian el agua, así Cleón no puede medrar si antes no se ve enturbiada la situación política. Los mejores ciudadanos velan por la paz y la tranquilidad públicas, los más depravados desean sobre todo la guerra y las rebeliones; $\|$ estos se afligen con la esperanza de paz, con las revueltas se regocijan y en medio del riesgo se sienten seguros, porque en el ámbito privado son unos degenerados, nocivos para el bien común, solo cifran sus esperanzas en las disensiones. Para estos la calma consiste en las sediciones, y la paz, en las revueltas; son los que no confían en obtener honores con la situación política serena, pero esperan conseguirlos con ella agitada. Cuando más resoluto y audaz es uno, más crédito e influencia alcanza en una rebelión.»

En la primera parte el autor aborda otro símil, también de signo político, el de los pescadores de anguilas; estos, como para capturarlas necesitan encenagar antes el agua ${ }^{33}$, son metáfora de los Cleones, $i$. e., de los demagogos y sediciosos, que enturbian el restańo de la ciudad en busca de su ganancia particular. La equivalencia simbólica entre los anguillarum venatores y el demagogo ateniense aparece por primera vez en los Equites de Aristófanes; en esta comedia satírica, Pafla-

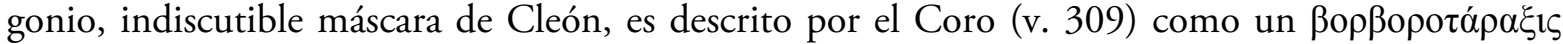
('removedor de fango') (Galy 1990); tal insulto, versos después (864-867), lo aclara el Morcillero

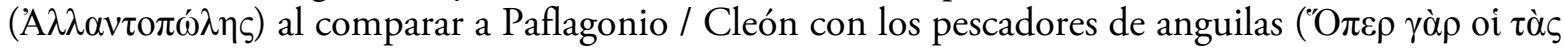

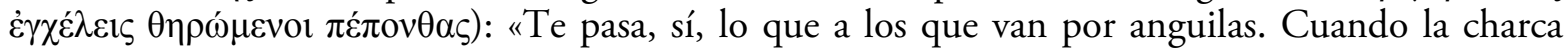

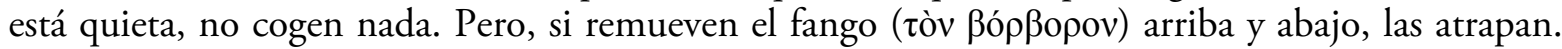
También sacas provecho tú, cuando revuelves la ciudad» (trad. Gil Fernández 1995).

Lebey toma la referencia, abreviándola, de los Collectanea (1. a ed. 1563) de J. Jacobus Manlius / Manlein ${ }^{34}$, quien en el "Quinto precepto» (De setionibus \& seditiosis), esboza la Pictura seditiosorum a partir del citado paso del comediógrafo griego ${ }^{35}$.

29 Tac., ann. 11, 17: qui privatim degeneres, in publicum exitiosi, nibil spei nisi per discordias habent. Cf. Lips. Polit. VI, IV (Waszink, ibid.).

30 Ps. Sall. orat. Phil. 7: Sed contra seditionibus omnia turbae sunt. Cf. Lips. Polit. VI, IV: Quibus quies in seditionibus, in pace turbae sunt (Waszink, ibid.).

31 Cic. Catil. 2, 19: honores, quos quieta republica desperant, perturbata consequi se posse arbitrantur. Cf. Lips. Polit. VI, IV (Waszink, ibid.).

32 Tac. ann. 1, 57, 1: Quanto quis audacia promptus, tanto magis fidus, rebusque motis potior habetur. Cf. Lips. Polit. VI, IV (Waszink 2004, 682).

33 Véase Ar. Eq. 864-867; Arist. HA 8, 592a.

${ }^{34}$ Este libro de lugares comunes, fuente habitual de Lebey (Antón 2014, 136), está compuesto tanto de extractos de las lecciones de Philip Melanchton (ex lectionibus D. Philippi Melanchthonis), a cuyo servicio estuvo Manlius (MacLean 2009, 121-123), como de las explicaciones de otros doctísimos varones (ex aliorum doctissimorum virorum relationibus).
35 Esto escribe Manlius (cito por la ed. de 1565, 279): Aristophanes [...] inquit: ut qui anguillas capiunt, prius turbant aquas [...] sic Cleon non potest crescere nisi prius turbata republica. Is Cleon homo turbulentissimus, multa scelerate fecit, fuit causa ut Athenienses amitterent oram Thraciae. Ita quemadmodum optimi quique semper paci ac tranquillitati reipublicae student: sic sceleratissimi maxime bellum et perturbationes amant ac desiderant. ("Aristófanes [...] dice: del mismo modo que quienes pescan anguilas antes enturbian el agua [...], así Cleón no puede medrar si antes no se ve enturbiada la situación política. Ese Cleón, hombre turbulentísimo, cometió muchas iniquidades y fue el responsable de que los atenienses perdieran la costa de Tracia. Por lo tanto, del mismo modo que los mejores ciudadanos siempre velan por la paz y tranquilidad pública, así los más depravados desean y persiguen sobre todo la guerra y las rebeliones»). Nótese la parte del texto que Lebey decidió omitir. 
La segunda parte es un ensamblaje de excerpta, en su mayoría de Tácito, copiados de los Politicorum libri o Política(s) (1589) de Justo Lipsio ${ }^{36}$, un libro de loci communes que nuestro emblematista utiliza con profusión (Antón 2014, 153-163).

En esta tupida red de citaciones, sorprende que Lebey omita los citados versos aristofánicos, posiblemente porque se habían convertido en un lugar común ampliamente divulgado. Como prueba, traigo aquí un pequeño pero significativo elenco de algunas obras que funcionaban como vivero de argumentos y de motivos simbólicos para los autores de los siglos XVI y XVII.

2.2.1. Empezaré por la Suda, y en la versión latina de Hieronimus Wolf, que es la que manejó

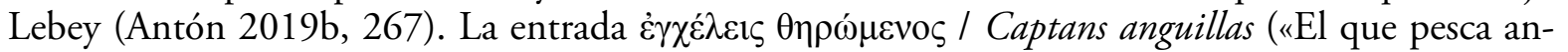
guilas»), siguiendo el loc. cit. de Aristófanes, dice: De ijs qui priuati emolumenti causa turbas concitant, quod anguillae turbata aqua \& commota capiantur ("[Se dice] de los que provocan revueltas para su lucro personal, pues las anguilas son capturadas con el agua revuelta y agitada») ${ }^{37}$.

\subsubsection{Erasmo repite el símil en el adag. III.VI.79 (n. ${ }^{\circ}$ 2579), Anguillas captare ${ }^{38}$ :}

'E $\gamma \chi \bar{\lambda} \lambda \varepsilon ı \varsigma ~ \theta \eta \rho \tilde{\sigma} \sigma \theta \alpha$, id est Anguillas venari, dicuntur, qui privati compendii causa cient tumultus. Inde ducta similitudine, quod, cum aqua stat immota, nihil capiunt, qui captant anguillas, cum vero sursum ac deorsum miscent ac perturbant aquam, ita demum capiunt. Quadrabit in eos, quibus tranquillo rei publicae statu nihil est emolumenti. Proinde seditiones gaudent exoriri, quo civitatis publicum malum in suum privatum vertant commodum.

«E $\gamma \chi \dot{\lambda} \lambda \varepsilon 1 \zeta$ $\theta \eta \rho \tilde{\alpha} \sigma \theta \alpha \mathrm{l}$, es decir, Pescar anguilas, se dice de los que para obtener una ganancia personal provocan tumultos. De ahí procede el símil de que, cuando el agua está inmóvil, no pescan nada los que capturan anguilas; pero, cuando revuelven y enturbian el agua, así al fin las pescan. Se acomodará a los que no obtienen ningún beneficio con la paz social. Se alegran de que surjan sediciones porque transforman el mal público de la ciudad en su beneficio personal.»

Erasmo transcribe (en griego y en latín) los versos aristofánicos (Eq. 864-867), equiparando

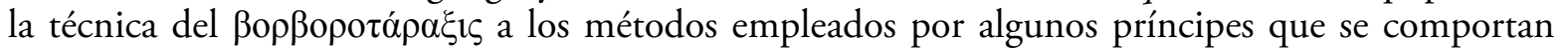
como tiranos:

Hanc artem heu nimium callent principes quidam, qui consilio tyrannico dissidia serunt inter civitates aut bellum aliquod alicunde suscitant, quo licentius mulctent miseram plebeculam et innocentissimorum civium fame suam expleant ingluviem.

¡Ay! Conocen bien esta técnica algunos príncipes, que con planes tiránicos siembran disensiones entre las ciudades o suscitan una guerra con cualquier pretexto, para depredar con más libertad al mísero populacho y saciar su propia voracidad, haciendo que pasen hambre los ciudadanos más honrados.»

36 Lo corroboran las variantes textuales de las notas bibliográficas que he recogido en las notas a pie de página (n. ${ }^{\text {os } 29-32) . ~}$
37 Cf. Suidae historica, 1. ${ }^{a}$ ed. Basilea, 1564 ,

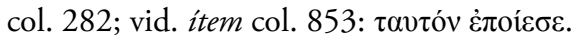

38 Saladin 2011, III 273. 
2.2.3. Alciato, posiblemente a la vista del adagio de su amigo Erasmo ${ }^{39}$, ideó el emblema In diuites publico malo («Sobre los que se enriquecen con las desgracias públicas»), que apareció por primera vez en la editio aldina (1546, 3r) [Fig. 4 $]^{40}$.

EMBLEM ITVMLIBELLVS. 3

In diuites publico malo.

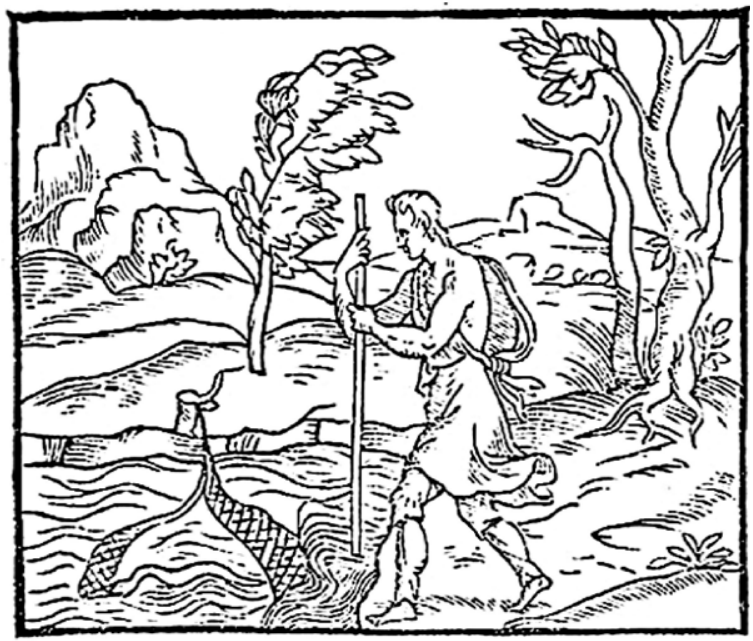

$$
\begin{aligned}
& \text { Anguillas quifguis captat, fi limpidd uerrat } \\
& \text { Flumina, fi illimes aufit adire licus, } \\
& \text { Caffus erit, lidet'q; operam . multum excitet ergo } \\
& \text { si crete, of uitreas pa!mula turbet aquas, } \\
& \text { Dines erit. fic ïs res publica turbida lucro eft, } \\
& \text { Quipace, arctati legibus, efuriunt. }
\end{aligned}
$$

Figura 4. A. Alciato, Emblematum Liber, Venecia, 1546, pág. 3r. URL: https://archive.org/details/itabnc-ald-00000151-001/mode/2up. No copyright.

$39 \mathrm{El}$ argumento remonta a Esopo, a la fábula «El pescador que batía el agua» (Chambry 1927, 27) y a Aristófanes (Eq. 864); y el lema, a Catón, De sumptu suo 173.1 (Meyer 1837), apud Cic. Cato 44: eos malo publico divites feci; véase Sciarrino 2010, 65.
40 Idéntico motivo en Camerario, Cent. IV, 45: Turbato flumine capta (Antón e. p.), que cita este emblema alciáteo y afirma que lo formó a partir de Esopo y Aristófanes. 
En el epigrama explica cómo han de capturarse las anguilas, reservando para el final la conlatio:

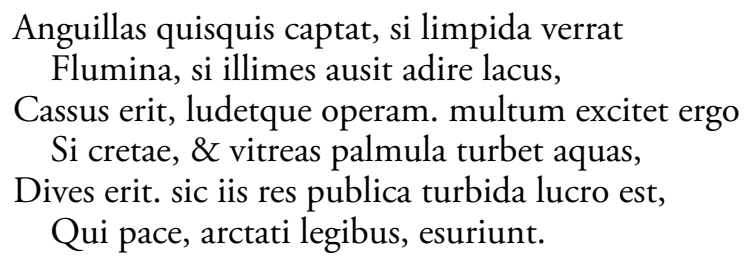

«Todo el que pesca anguilas, si echa la red en un río de límpida corriente, si se atreve a entrar en un diáfano lago, volverá de vacío y perderá el tiempo. Pero, si remueve mucho el limo y agita las aguas cristalinas con un remo, se hará rico. Así son los que se lucran de las revueltas públicas y que, coartados por las leyes, pasan necesidad en tiempo de paz.»

2.2.4. Pierio Valeriano, a cuyos Hieroglyphica (1556) acude a menudo Lebey (Antón 2019b, 27) ${ }^{41}$, en el Lib. XXIX, capítulo De anguilla (fol. 210E-F), enuncia la técnica de pescar anguilas como jeroglífico de los que se enriquecen durante las guerras civiles (Per discordias civium locupletatus $)^{42}$ :

Hominem uero ex ciuilibus seditionibus \& tumultuosis discordijs crescentem, reque auctum significantes, eum in Anguillarum uenatione occupatum effingebant. Aqua siquidem quieta limpidaque earum captura nulla propemodum, magna uero si perturbetur. Quare piscatores tempestates obseruant, lacuumue aut fluminum turbationes, unde aqua limosa fiat, magnas inde capturas paruo negotio consequentes.

"Cuando querían significar el hombre que medra aprovechando las guerras civiles y las tumultuosas discordias, y que prospera con ello, lo representaban atareado pescando anguilas. Si en efecto el agua está serena y limpia, casi no obtienen ninguna, pero muchas si está revuelta. Por eso, los pescadores observan las borrascas que agitan el agua de los ríos y lagos haciendo que se vuelva fangosa y entonces consiguen grandes capturas con poco esfuerzo.»

2.2.5. El último representante de este manipulus es el médico Conrad Gesner, ya nombrado. En el vol. IV de sus Historiae animalium (1558), al tratar De anguillis reúne el loc. cit. de Equites $(47 \$ \mathrm{E})$, el adagio erasmiano y el emblema alciáteo $(56 \$ \mathrm{H})^{43}$ :

Ex eodem fonte [in marg.: Aristof. Equit.] manasse puto id quod prouerbij loco apud nos iactatur, Piscari in aqua turbida, quod dicitur in eos qui ex litibus, bellis, seditionibus: denique ex quietis uel priuatae uel publicae perturbatione magnos fructus percipiunt, ac maxime hinc facultates suas augent.

41 Sin embargo, Lebey no pudo servirse de Valeriano para el emb. XXIII porque no dedica a la salamandra ningún jeroglífico que encaje con el simbolismo político aquí tratado.

42 Después cita, y en este orden, a Aristóteles, Aristófanes y Cicerón: Rem tradit Aristoteles [HA 8, 529a] \& plerique alij, tum Aristophanes, Equitibus [863-864], historiam luculenter admodum explicat, [...] Quam senten- tiam Cicero sibi desumpsit, oratione in Catilinam secunda [Catil. 2, 9, 19]: Honores quos quieta Republica desperant, turbata consequi se posse arbitrantur. Las edd. modernas prefieren perturbata en vez de turbata.

43 Aclararé que las secciones de cada animal se indican con letras de la A a la $\mathrm{H}$. La E incluye la captura y muerte de las anguilas; la $\mathrm{H}$ abarca anécdotas, alegorías y simbolismos de las mismas. 
«De esa misma fuente, según creo, procede la expresión que, como proverbio, circula entre nosotros, Pescar en agua revuelta ${ }^{44}$. Eso se dice de los que, a consecuencia de las contiendas, las guerras y las rebeliones, en suma, de perturbar la paz privada o pública, obtienen grandes ganancias, y con ello incrementan muchísimo sus oportunidades.»

\section{APÉNDICE: EXEMPLA HISTÓRICOS}

La paráfrasis se prolonga con una amalgama de pasajes griegos (siempre en versión latina ${ }^{45}$ ) y latinos, presididos por dos paremias: In dissidio vel Androclides fit Imperator. In summis difficultatibus etjam Carcinus honoratur («En una guerra civil incluso Andróclides es nombrado general. En las dificultades extremas también el cangrejo recibe honores») ${ }^{46}$, que coinciden con la versión de la Suda de H. Wolf (1564, col. 296). Con ambos adagios, que hacen de bisagra entre las dos primeras secciones y esta tercera, se llega al final de la página, por lo que Lebey remite al lector a buscar la continuación en la antepenúltima página del libro, en un addendum bajo la letra C (Sequentia verba quaere pag. antepen. sub litera C.). Allí prosigue la explanatio con un pasaje de Plutarco (Nic. 11, 3 [530D]), tomado de la versión latina del humanista holandés H. Cruser (Antón 2014, 137), alterando el orden de las frases ${ }^{47}$ :

Et Hyperdocus [Hyperbolus] ille Perithaedes [Perithoedes] ${ }^{48}$ Athenis homo ex nullis audaciam sumens opibus, sed ex audacia ad opes promotus, factus [ex] eo quem in ciuitate habebat honorem ignominia ciuitatis, cum nempe distracta tunc in duas factiones ciuitas audacissimis et versutissimis locum daret.

«Y aquel famoso Hipérbolo de Peritedes, un ateniense que no adquirió osadía como consecuencia de su poder, sino que gracias a su osadía llegó al poder; por eso, la reputación que aquel tenía en la ciudad se tornó en un desdoro para la ciudad cuando, por estar entonces la ciudad escindida en dos facciones, cedió ante los más audaces y taimados.»

${ }_{44}$ De ahí los refranes castellanos «Pescar en río revuelto» $\mathrm{y}$ «A río revuelto, ganancia de pescadores» (Herrera Vázquez 2017), con equivalencia en muchas otras lenguas (Arthaber 1995, 1066; Flonta 2012, 1008).

45 Lebey declara que no se le permitió incluir citas griegas (Antón 2014, 132).

46 Esas paremias, con variantes, las cita Erasmo, adag. II.II.91 (1191) (Saladin 2011, II 136): In seditione vel Androclides belli ducem agit / Fert rebus desperatis et cancer honorem; adag. II.1.12 (n. ${ }^{\circ}$ 1012) (Saladin 2011, II 24): In seditione etiam Androclides abjectissimus belli ducem agat.

47 Cito por la ed. de Venecia, 1572, 259a [in marg.: Hyperbolus], donde dice: Siquidem distracta \& tunc in duas ciuitatis factiones dedit audacissimis et versutissi- mis locum, in quibus Hyperbolus erat Perithoedes, homo ex nullis audaciam sumens opibus, sed ex audacia ad opes promotus: factusque ex eo, quem habebat in ciuitate honorem, ignominia ciuitatis. ("Puesto que la ciudad, entonces escindida en dos facciones, cedió ante los más audaces y taimados, entre los que se encontraba Hipérbolo de Peritedes, un hombre que no adquirió osadía como consecuencia de su poder, sino que gracias a su osadía llegó al poder; por eso, la consideración de que gozaba en la ciudad acabó siendo una ignominia para la ciudad».)

${ }^{48}$ En Aristófanes (Eq. 846-847) aparece la primera mención de Hipérbolo, un rico fabricante de lámparas que sucedió a Cleón; fue asesinado por los oligarcas en el año 411 (Plácido 2012). 
Enhebra dos loci de Claudiano desgajados del carmen XX, in Euthropium:

Sic in rerum tumultu pro tempore Pars humili de plebe duces, pars compede suras Cruraque signati nigro renitentia [liventia] ferro Iura regunt ${ }^{49}$, coquus hic, hic leno, hic sępius emptus, Alter ad Hispanos nutritus verna parentes. ${ }^{50}$

«Así, en una sublevación, según las circunstancias, una parte, procedente de la baja plebe, alcanza el rango de general; otra parte, con sus pantorrillas señaladas por los grilletes y sus piernas amoratadas por el negro hierro, administra justicia; uno, un cocinero; otro, un proxeneta; uno, comprado reiteradas veces; el otro, criado como esclavo nativo en un hogar hispano.»

De la mano de Claudiano va Plutarco con palabras del inicio de la Lysandri cum Sylla comparatio $(475 \mathrm{~F})$, en la traducción citada de Cruser $(1572,379)^{51}$ :

Et quid iam mirum si dominatus Sylla est quum Glauciae \& Saturnini exigerent Vrbe Metellos, Consulum filij in concione occiderentur, auro \& argento arma pararent, milites redimentes, ferro \& igni leges figerent, vim adhibentes repugnantibus?

«No hay que admirarse de que Sila se hubiera hecho con el poder, cuando los Glaucias y Saturninos expulsaban de la ciudad a los Metelos, cuando los hijos de los cónsules eran asesinados en las asambleas públicas, cuando con oro y plata conseguían armas, comprando a los soldados, cuando a hierro y fuego se promulgaban las leyes, empleando la violencia contra los oponentes.»

No creo que sea fortuito que Lebey haya decidido formar el residuum de la paráfrasis con estos exempla. Es presumible que percibiera la semejanza entre todos esos personajes (los plebeyos que llegaron a ser generales y los siervos que acabaron ejerciendo de jueces, los demagogos griegos y romanos) con los hombres indignos e infames que, en la Francia de su tiempo, fomentaban los disturbios y, por mor de ellos, alcanzaban prestigio y poder. No olvidemos que nuestro autor estuvo a punto de perder la vida en las matanzas de hugonotes que se produjeron por toda Francia en la noche de san Bartolomé (23 al 24 de agosto de 1572) y que a él lo sorprendieron en Valence; logró salvarse, al igual que otros compañeros de estudios, gracias a la mediación de su maestro Jacques $\mathrm{Cujas}^{52}$. Las turbulencias que zarandearon la sociedad francesa del siglo XVI ponen el colofón a este emblema, cuyo estudio concluiremos trasladando su argumento al mundo actual.

49 Claud. carm. 20, 342-344: Pars humili de plebe duces, pars compede suras / Cruraque signati nigro liventia ferro / Iura regunt.

50 Claud. carm 20, 351-353: Hic coquus, hic leno, defessi verbere terga, / servitio, non arte, pares, hic saepius emptus, / Alter ad Hispanos nutritus verna parentes. («Uno, un cocinero; otro, un proxeneta, con sus espaldas sañaladas por los azotes; iguales en esclavitud, no en habilidad; uno, comprado reiteradas veces, el otro, criado como esclavo nativo en un hogar hispano».) Adviértase la frase de la que ha prescindido Lebey.
51 Et quid miri, si dominatus Sylla est: cum Glauciae \& Saturnini exigerent vrbe Metellos, consulum filij in concione occiderentur, auro \& argento arma pararent, milites redimentes, ferro \& igni leges figerent, vim adhibentes repugnantibus). Lebey modifica ligeramente el inicio: iam mirum por miri.

52 Cullière 1991, 10; Antón 2014, 119; Meerhoff 2017, 49 . 


\section{EPÍLOGO}

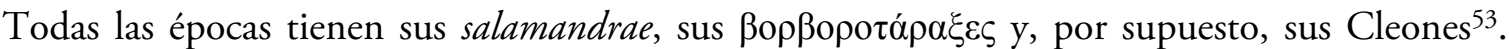
También la nuestra. Y, a día de hoy, a juzgar por los testimonios que he podido recabar, el «Cleón» mundialmente más famoso e influyente es Donald Trump ${ }^{54}$.

Los paralelismos entre el demagogo ateniense y el político norteamericano han sido puestos de realce por muchos estudiosos, si bien el más extenso y de más calado científico se debe a Bernard J. Dobski (2018), profesor de ciencias políticas en Assumption College (Worcester, MA), quien subtitula su trabajo con este sugerente quiasmo (en cursiva en el original): "Athenian» Trump, "American» Cleon. Pero especialmente es en Google donde se constata una retahíla de columnas, blogs, artículos, etc. que establecen llamativas semejanzas entre ambos personajes, destacando en particular las contribuciones de los profesores Chris Máchi (La Trobe University) ${ }^{55}$, Kevin Morrell (University of Warwick, UK) ${ }^{56}$ y Elizabeth Markovits (Mount Holyok College) ${ }^{57}$, para no alargar más la lista $^{58}$.

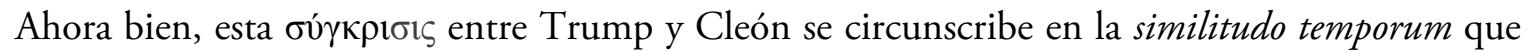
muchos espíritus aprecian entre la Atenas del siglo v a.C. y los Estados Unidos del siglo xxi, y eso es posible porque, por decirlo a la manera de Tácito (hist. 2, 95, 3), magis alii homines quam alii mores.

\section{BibliografíA}

Adams, A., 2007, «La conception et l' édition des livres d' emblèmes dans la France du Xvi siècle. Une problématique collaboration entre un auteur et un éditeur», Littérature 145/1, 10-22.

Adams, A., S. Rawles \& A. Saunders, 1999-2002, A Bibliography of French Emblem Books of the Sixteenth and Seventeenth Centuries (BFEB), Genève: Droz, 2 vols.

Alciato, A., 1546, Emblematum Libellus, nuper in lucem editus, Venetiis: apud Aldi filios.

Antón, B., 2012, "Diarii omnes. La huella de Juan Luis Vives en los Symbola et Emblemata de Joaquín Camerario", SPhV 14, 157-170.

Antón, B., 2014, «Los Emblemata (Frankfurt, 1596) de Dionysius Lebeus Batillius: Clasicismo, Neoestoicismo, Calvinismo", Janus. Estudios sobre el Siglo de Oro 3, 115-176.

Antón, B., 2016, «Andrea Alciato, dux et praeses del género emblemático», Insula 833, 18-22.

Antón, B., 2019a, "La (vera) Amicitia en los Emblemata (1596) de Denis Lebey de Batilly», en: V. Alfaro, V. E. Rodríguez, G. Senés (eds.), Studia Classica et Emblematica caro magistro Francisco J. Talauera Esteso dicata, Zaragoza: Libros Pórtico, 107-157.

53 Así, v. gr., Droysen $(1837,287)$ apunta la relevancia histórica de Cleón, similar a la del sanguinario Robespierre o al salvaje Mario (des blutingen Robespierre oder des wilden Marius).

54 También ha sido comparado con Julio César, con Publio Clodio o incluso con Nerón. Así, J. Connolly (City University of New York): «The Romans Tried to Save the Republic From Men Like Trump. They Failed». The Village Voice, 17 Jan. 2017; P. Freeman (Seaver College, Pepperdine University): "Meet the Trump of Ancient Rome, a populist demagogue who helped bring down the Republic». The Haffington Post, 12 Apr. 2016;
J. Webster (Eugene Lang College): «Donald Trump: the Modern-day Neron ready to burn down America». The Gardian, 10 Jun. 2016.

55 «Can we learn from Thucydides' writings on the Trump of ancient Athens?». The Conversation, $7 \mathrm{Au}$ gust, 2016.

56 «Before There Was Trump, There Was Cleon». White House Chronicle, January 25, 2017.

57 «Trump "tells it like it is». That's not necessarily a good thing for democracy». Washington Post, March 4, 2016.

58 Más referencias en Dobski 2018, 90-91 n. 2 y 9. 
AnTón, B., 2019b, «La representación simbólica de la paz: la disputa por el patronazgo de Atenas en los Emblemata (eds. 1596 y 1600) de Denis Lebey de Batilly», Euphrosyne 47, 247-269.

Antón, B., e. p., "La representación zoológico-simbólica de los sediciosos y agitadores en los Symbola et Emblemata de Joaquín Camerario", Anuario de Estudios Filológicos (AEF) 44.

ARthaber, A., 1995, Dizionario comparato di proverbi e modi proverbiali in sette lingue: italiana, latina, francese, spagnola, tedesca, inglese, greca antica, Milano: U. Hoepli.

Biederbick, M. C., 2017, «Tradition and Empirical Observation-Nature in Giovio's and Symeoni’s Dialogo dell'Imprese from 1574», en: Karl A.E. Enenkel, J. Smith (eds.), Emblems and the Natural World, Leiden-Boston: Brill, 271-320.

Boissard, J. J., 1593, Emblematum Liber. Ipsa Emblemata ab Auctore delineata: a Theodoro de Bry sculpta, Francofurti ad Moenum: De Bry.

Busolt, G., 1904, Griechische Geschichte bis zur Schlacht bei Chaeroneia, III.2. Gotha: Perthes.

Camerario, J., 1604 [1605], Symbolorum et Emblematum ex aquatilibus et reptilibus desumptorum Centuria Quarta a Joachimo Camerario Medi[co] Norimberg[ensi] coepta: absoluta post eius obitum Ludouico Camerario: [s. l.]: [s. n.].

Canfora, L., 2016, Tucidide, la menzogna, la colpa, l'esilio, Bari: Laterza \& Figli.

Canfora, L., 2017, Cleofonte deve morire: Teatro e politica in Aristofane, Bari: Laterza.

Chambry, E., 1927, Esope. Fables, Paris: Les Belles Lettres.

Choné, P., 2007, «Les cas singulier des emblèmes en Lorraine aux xvıe et xvıre siècles», Littérature 145/1, 79-90.

Coustau, P., 1555, Pegma cum narrationibus philosophicis, Lugduni: apud Matthiam Bonhomme.

Cullière, A., 1991, «Un pamphlet autographe de Denis Lebey de Batilly (1590)», BHR 53/1, 7-32.

Doвsкi, B., 2018, «Democracy, Demagogues, and Political Wisdom: Understanding Trump in the Wake of Thucydides' History», en: A. Jaramillo Torres, M. B. Sable (eds.), Trump and Political Philosophy: Leadership, Statesmanship, and Tyranny, London: Palgrave Macmillan, 75-93.

Droysen, J. G., 1837, Des Aristophanes Werke, vol. II, Berlin: Veit.

Erasmo, D., 1514, Parabolarum, siue Similium Liber, [Colofón] Argentorati: ex Aedibus Schurerianis.

Flonta, T., 2012, A Dictionary of English and Romance Languages Equivalent Proverbs, Tasmania, Australia: DeProverbio.com.

Foster, E., 2017, «Aristophanes's Cleon and Post-Peloponnesian War Athenians: Denunciations in Thucydides", Historia Supplement 6, 129-152.

Galy, J. M., 1990, "Les borborotaraxes. Politique et politiciens dans la comédie grecque des vème et Iv ${ }^{\mathrm{ème}}$ siecles», en: J. Granarolo, M. Biraud (eds.), Hommage à René Braun I: De la préhistoire à Virgile: philologie, littératures et histoires anciennes, Nice-Paris: Les Belles Lettres.

Gesner, C., 1554, Historia animalium Liber II, De quadrupedibus oviparis, Tiguri: apud Christophum Froschoverum.

Gesner, C., 1558, Historiae animalium liber IIII, qui est de piscium et aquatilium animantium natura, Tiguri: apud Christophum Froschoverum.

García Arranz, J. J., 1990, «La salamandra: distintas interpretaciones gráficas de un mito literario tradicional», Norba: Revista de Arte 10, 53-68.

Gil Fernández, L., 1995, Aristófanes. Comedias I: Los Acarnienses - Los Caballeros, Introducción, traducción y notas, Madrid: Gredos.

Gladhill, B., 2016, Rethinking Roman Alliance: A Study in Poetics and Society, Cambridge: Cambridge U. P.

Graham, D. (ed.), 2001, An Interregnum of the Sing. The Emblematic Age in France. Essays in Honour of Daniel S. Rusell, Glasgow: Glasgow University.

Grote, G., 1854, History of Greece, London: John Murray.

Gustainis, J. J., 1990, «Demagoguery and Political Rhetoric: A Review of the Literature», Rhetoric Society Quarterly 20/2, 155-161.

Hayaert, V., 2005, «Pierre Coustau's Le Pegme (1555): Emblematics and Legal Humanism», Emblematica. An Interdisciplinary Journal for Emblem Studies 14, 55-99. 
Hayaert, V., 2008, «Mens emblematica» et humanisme juridique: le cas du «Pegma cum narrationibus philosophicis» de Pierre Coustau (1555), Genève: Droz.

Hendrikx, S., 2017, «Ichthyology and Emblematics in Conrad Gesner's Historia piscium and Joachim Camerarius the Younger's Symbola et emblemata», en: K. A. E. Enenkel, P. J. Smith (eds.), Emblems and the Natural World, Leiden-Boston: Brill, 184-226.

Henkel, A. \& A. Schöne, 2013, Emblemata: Handbuch zur Sinnbildkunst des XVI. und XVII. Jahrhunderts, Stuttgart: Metzler.

Herrera Vázquez, M., 2017, "Contribución al estudio del refrán A río revuelto, ganancia de pescadores», en: F. Toro Ceballos (ed.), Juan Ruiz, Arcipreste de Hita, y "El libro de Buen Amor» [...] Homenaje a Joseph T. Snow, Alcalá la Real: Ayuntamiento, 147-161.

Lafargue, Ph., 2013, Cléon, le guerrier d'Athéna, Bordeaux: Ausonius.

Lebey, D., 1596, Emblemata a Jano Jac. Boissardo Vesuntino delineata sunt, et a Theodoro de Bry sculpta, et nunc recens in lucem edita, Francofurti ad Moneum: De Bry.

Lebey, D., 1600, Emblemata. [...] Vna cum Appendice nonnullorum carminum. Editione Voegeliniana. Dionysii Lebei Batillii, et Aliorum Aliquot Poematia, Heidelbergae: [Voegelin].

Lipsio, J., 1589, Politicorum sive civilis doctrinae libri sex, Antuerpiae: apud Christophorum Plantinum. Vid. J. WASZINK.

MacLean, I., 2009, Learning and the Market Place: Essays in the History of the Early Modern Book, Leiden: Brill.

Manlius, J., 1565, Locorum communium collectanea a Iohanne Manlio per multos annos tum ex lectionibus D. Philippi Melanchthonis, tum ex aliorum doctissimorum virorum relationibus excerpta, Francofurti ad Moenum: per Petrum Fabricium.

Mann, Ch., 2007, Die Demagogen und das Volk. Zur politischen Kommunikation im Athen des 5. Jahrhunderts v. Chr. [Series: Klio], Berlin: Akademie.

Margolin, J.-C. (ed.), 1975, Parabolae sive similia, en: Opera Omnia Desiderii Erasmi Roterodami Recognita et Adnotatione Critica Instructa Notisque Illustrata, Ordinis Primi Tomus Quintus, Amsterdam-Oxford: North-Holland Publishing Company.

Matthioli, P. A., 1554, Commentarii, in libros sex Pedacii Dioscoridis Anazarbei, De medica materia, Venetiis: in officina Erasmiana, apud Vincentium Valgrisium.

Meerhoff, K., 2017, «Éloge de la poésie dans les Emblemata (1596) de Lebey de Batilly», RHR 85, 47-94.

Meyer, H., 1837, Oratorum romanorum fragmenta ab Appio inde Caeco usque ad Q. Aurel. Symmachum, editio parisina auctior et emendatior, curis Fr. Dübner, Parisiis: Bourgeois-Maze.

Morwood, J., 2009, «Euripides and the Demagogues», CQ 59/2, 353-363.

Paladini, M.a L., 1958, "Considerazioni sulle fonti della storia di Cleone», Historia. Zeitschrift für Alte Geschichte $7 / 1,48-73$.

PAPY, J., 2003, "Joachim Camerarius's Symbolorum et Emblematum Centuriae Quatuor: From Natural Sciences to Moral», en: Karl A. E. Enenkel, Arnoud S. Q. Visser (eds.), Mundus Emblematicus, Brepols: Turnhout, 201-234.

Plácido, D., 2012, "Aristófanes como fuente de la historia social, Acarnienses 1-42», Pecia Complutense $17 / 9,1-13$.

Plutarco, 1572, Plutarchi Queronei [...] Vitae comparatae Illustrium virorum, Graecorum \& Romanorum, [...] Hermanno Cruserio [...] interprete, Venetiis: Hieronymus Scotus.

Russell, D., 1985, The Emblem and Device in France, Lexington, KY: French Forum Publishers.

Saladin, J.-C. (ed.), 2011, Les Adages d'Érasme, Paris: Les Belles Lettres, 5 vols.

Saldutti, V., 2014, Cleone un politico ateniese, Bari: EdiPuglia.

Saunders, A., 1988, The Sixteenth-Century French Emblem Book: A Decorative and Useful Genre, Genève: Droz.

SAUnders, A., 2001, "Quis, quid, ubi, quibus auxiliis, cur, quomodo, quando? or: The curious case of Pierre Coustau's Pegma», en: D. Graham (ed.), An Interregnum of the Sing. The Emblematic Age in France, Glasgow: Glasgow University, 29-48. 
Sciarrino, E., 2010, «Roman Oratory Before Cicero: The Elder Cato and Gaius Gracchus», en: W. Dominik, J. Hall (eds.), A Companion to Roman Rhetoric, Oxford-Malden-Carlton: Wiley-Blackwell, 54-65.

SudA, 1564, Suidae historica, caeteraque omnia quae ulla ex parte ad cognitionem rerum spectant, [...] Nunc primum [...] opera uero ac studio Hiero. Wolfij in Latinum sermonem conuersa, Basileae: apud Ioannes Oporinum \& Hervagium.

Thibaudet, A., 1922, La campagne avec Thucydide, Paris: Nouvelle Revue Française.

Valeriano, P., 1556, Hieroglyphica, sive De sacris Aegyptiorum literis commentarii, Basileae: Palma Ising[rini].

Velo-Antón, G. \& D. Buckley, 2015, «Salamandra común - Salamandra salamandra», en: S. A. Martínez, I. Solano (eds.), Enciclopedia Virtual de los Vertebrados Españoles, Madrid: Museo Nacional de Ciencias Naturales. http://digital.csic.es/bitstream/10261/126388/6/salsal_v1.pdf [Consulta 27-10-2020].

Waszink, J., 2004, Justus Lipsius, Politica. Six books of Politics or Political Instruction (edition, introd. and transl.), Assen: Royal van Gorcum. 